\section{AVALIAÇÃO DO NÍVEL DE INFORMAÇÃO SOBRE HANSENÍASE DOS AGENTES COMUNITÁRIOS DE SAÚDE}

\author{
Assessment of the level of information on leprosy by community \\ health workers
Evaluación del nivel de información delos agentes comunitarios de salud sobre la lepra

\section{RESUMO}

Objetivo: Avaliar o nível de informação dos Agentes Comunitários de Saúde (ACS) sobre a hanseníase e os aspectos como diagnóstico, cura, tratamento e transmissão. Métodos: Tratase de uma pesquisa quantitativa, descritiva e transversal. Para a coleta de dados utilizou-se um questionário com perguntas objetivas divididas em tópicos sobre a doença: aspectos gerais (bloco 1), diagnóstico (bloco 2), transmissão (bloco 3) e tratamento (bloco 4) da hanseníase, aplicado a 43 ACS de Cocal, Piauí, entre janeiro e março de 2016. Os participantes tinham como opções de respostas: sim, não e não sei responder. Resultados: Foi observado um nível de conhecimento "bom" para o bloco 3, "regular" para os blocos 1 e 4 e "ruim" para o bloco 2. Quando analisado o panorama geral, o conhecimento dos ACS foi considerado regular. Conclusão: Os resultados obtidos nesta pesquisa evidenciaram um nível de informação aquém do esperado para os ACS sobre os principais aspectos da hanseníase, fazendo-se necessário que maior atenção seja dada na educação permanente em saúde desse profissional.

Descritores: Hanseníase; Agente Comunitário de Saúde; Saúde Pública.

\section{ABSTRACT}

Objective: To assess the level of information of Community Health Workers (CHW) regarding leprosy and aspects such as diagnosis, cure, treatment and transmission. Methods: This is a quantitative descriptive cross-sectional study. Data were collected using a questionnaire with closed-ended questions divided into topics about the disease - general aspects (block 1), diagnosis (block 2), transmission (block 3) and treatment (block 4) of leprosy - applied to 43 CHWs from Cocal, Piaui from January to March 2016. Participants had to choose between the response options: yeas, no or do not know. Results: the level of knowledge was "good" in block 3, "regular" in blocks 1 and 4 and "poor" in block 2. Considering the general overview, CHWs knowledge was considered fair. Conclusion: The results obtained in this study showed a performance below expectations for CHWs regarding the main aspects of leprosy, pointing out that more attention should be given to the continuing health education of this professional.

Descriptors: Leprosy; Community Health Worker; Public Health.
Artigo Original
1) Hospital Universitário Presidente Dutra - Universidade Federal do Maranhão - HUUFMA - São Luis (MA) - Brasil

2) Universidade Federal do Ceará - UFC Sobral (CE) - Brasil

3) Universidade Federal de Minas Gerais UFMG - Belo Horizonte (MG) - Brasil
Recebido em: 20/06/2016 Revisado em: 15/07/2016 Aceito em: 22/09/2016 


\section{RESUMEN}

Objetivo: Evaluar el nivel de información de los Agentes Comunitarios de Salud (ACS) sobre la lepra y sus aspectos como el diagnóstico, la cura, el tratamiento y la transmisión. Métodos: Se trata de una investigación cuantitativa, descriptiva y transversal. Para la recogida de datos se utilizó una encuesta con preguntas tipo test divididas en tópicos sobre la enfermedad: los aspectos generales (bloque 1), el diagnóstico (bloque 2), la transmisión (bloque 3) y el tratamiento (bloque 4) de la lepra que fue aplicada a 43 ACS de Cocal, Piaui, entre enero y marzo de 2016. Los participantes tenían como opciones de respuestas: sí, no y no sé contestar. Resultados: Se observó un nivel de conocimiento "bueno" para el bloque 3, "regular" para los bloques 1 y 4 y "malo" para el bloque 2. El conocimiento de los ACS fue considerado regular tras analizar el panorama general. Conclusión: Los resultados de esta investigación evidenciaron un nivel de información mayor de lo que se esperaba de los ACS sobre los principales aspectos de la lepra, señalando la necesidad de más atención para la educación permanente en salud de ese profesional.

Descriptores: Lepra; Agentes Comunitarios de Salud; Salud Pública.

\section{INTRODUÇÃO}

A hanseníase é uma doença crônica proveniente da infecção causada pelo Mycobacterium leprae, com alta capacidade de infectar grande número de indivíduos. A doença é caracterizada pela alta infectividade, no entanto poucos adoecem devido à baixa patogenicidade do bacilo ${ }^{(1)}$.

A hanseníase é considerada um problema de saúde pública nos países em desenvolvimento. As maiores taxas de acometimento da hanseníase são observadas em países da faixa intertropical e subdesenvolvidos economicamente ${ }^{(2-4)}$. Os maiores índices de notificação são de países da Ásia e Américas, onde o Brasil se destaca por ser o segundo país em número de casos no mundo ${ }^{(5)}$. A Organização Mundial da Saúde (OMS) classifica a hanseníase em duas formas, paucibacilar e multibacilar, sendo o acometimento neural a principal razão de estigma aos acometidos pelo bacilo de Hansen $^{(4,6)}$.

No Brasil, as regiões Norte e Nordeste são responsáveis pela maior incidência e prevalência de casos. De acordo com o Ministério da Saúde (MS), o estado do Piauí se destaca dentre os estados do Nordeste por ser o segundo com maior prevalência e o quinto com maior incidência de casos da doença ${ }^{(7)}$.

No Brasil, é necessário intensificar as ações de vigilância da hanseníase, voltadas especialmente à maior efetividade no diagnóstico e tratamento da doença, sobretudo nas regiões que apresentam maior concentração dos casos no país ${ }^{(8)}$. A principal estratégia do MS é a integração das ações de diagnóstico e tratamento da doença na atenção básica. Desta forma, as equipes da Estratégia Saúde da Família (ESF), Agentes Comunitários de Saúde (ACS) e todas as unidades do Sistema Único de Saúde (SUS), passam a integrar a rede de atendimento ao paciente, facilitando o acesso universal ao diagnóstico e tratamento ${ }^{(7)}$. Além disso, é importante o contínuo aperfeiçoamento dos sistemas de informação, atividade fundamental para garantir o adequado monitoramento da situação epidemiológica da hanseníase no Brasil, tendo em vista o alcance da meta de eliminação da doença como problema de saúde pública ${ }^{(8,9)}$.

A implantação SUS deu origem à criação de estratégias importantes de interiorização e promoção de saúde, além de mudança na orientação das políticas públicas de saúde. Nesse contexto, destaca-se a prevenção em saúde e o surgimento da ESF, além de profissões de interação entre o sistema de saúde e a comunidade, como os $\mathrm{ACS}^{(10)}$. Todas essas medidas visam evitar que os casos de doenças se agravem, sendo solucionados na atenção básica, e que a saúde seja levada até o usuário no âmbito familiar, tratando a família como um todo ${ }^{(11,12)}$.

A partir de então, houve o processo de descentralização do atendimento à hanseníase com a capacitação dos profissionais de saúde da família: ACS, técnicos de enfermagem, médicos e enfermeiros de família. A finalidade desta interiorização seria o atendimento dos casos nas unidades locais, além da promoção de atividades de educação em saúde. Tal fato resultou numa maior efetividade da descentralização das ações de controle da doença, com consequente aumento do coeficiente de detecção e menor abandono do tratamento ${ }^{(13)}$.

As campanhas de combate contra a hanseníase visam fortalecer as ações de vigilância epidemiológica e promoção da saúde, principalmente a educação permanente dos profissionais da saúde envolvidos na identificação de casos novos e acompanhamento dos pacientes em tratamento ${ }^{(3,14,15)}$. Destaca-se o profissional ACS responsável pelo elo entre Unidade Básica de Saúde (UBS) e a comunidade, tido como um disseminador de informações e responsável por identificação de novos $\operatorname{casos}^{(16,17)}$.

$\mathrm{O}$ ACS atua em conjunto com a equipe multidisciplinar no acompanhamento dos pacientes em tratamento de hanseníase, no intuito de reduzir as recidivas, identificação de contatos e casos novos ${ }^{(18)}$. Por meio das visitas domiciliares, o agente orienta o paciente atingido por hanseníase quanto ao autocuidado, administração adequada da medicação e visitas regulares a UBS para avaliação pela equipe médica e de enfermagem do correto andamento do tratamento $^{(12,15,18)}$. 
Um adequado nível de informação dos ACS sobre aspectos como diagnóstico, transmissão e tratamento da hanseníase promove dispersão de informações úteis aos usuários para redução de casos e recidivas ${ }^{(3,12,14)}$. O objetivo desse artigo foi avaliar o nível de informação dos ACS sobre a hanseníase e os aspectos como diagnóstico, cura, tratamento e transmissão.

\section{MÉTODOS}

O presente estudo consiste em uma análise quantitativa, descritiva, transversal realizado no município de Cocal - PI no período entre janeiro a março de 2016. O município conta com 14 equipes de Estratégia Saúde da Família (ESF), distribuídas entre zona urbana e rural, composta por um total de $68 \mathrm{ACS}$, além de médicos, enfermeiros e técnicos de enfermagem ${ }^{(19)}$.

Foram inclusos no estudo os ACS vinculados à Secretaria Municipal de Saúde (SMS) do município. Além disso, o preenchimento completo do questionário e a obtenção do Termo de Consentimento Livre e Esclarecido (TCLE) também foram critérios de inclusão. Foram excluídos os questionários incompletos e ou questionários de profissionais que não assinaram o TCLE, assim como os ACS que não se encontravam atuantes no momento do estudo.

Um questionário contendo 32 questões, contemplando tópicos sobre aspectos gerais, diagnóstico, transmissão e tratamento da hanseníase foi aplicado aos ACS na tentativa de mensurar o nível de informação desses profissionais. Os participantes tinham como opções de respostas: sim, não e não sei responder. Aplicou-se o questionário em uma sala reservada da UBS, sem acesso a meios que possibilitassem auxílio para resolução das questões e sob a supervisão de um pesquisador previamente treinado.

Para análise dos dados, foi adotada a seguinte escala elaborada pelos autores, com base no total de respostas corretas obtidas por meio do questionário:

Tabela I - Escala de acertos para mensuração do nível de informação dos Agentes Comunitários de Saúde (ACS) sobre hanseníase. Cocal, Piauí, 2016.

\begin{tabular}{lc}
\hline Nível de conhecimento & Total de acertos $\mathbf{( \% )}$ \\
\hline Ótimo & $90-100$ \\
Muito bom & $80-89$ \\
Bom & $70-79$ \\
Regular & $60-69$ \\
Ruim & $50-59$ \\
Muito ruim & Abaixo de 50 \\
\hline
\end{tabular}

Os dados foram tabulados no Office Excel versão 2013. A análise estatística empregada foi do tipo descritiva baseada na leitura dos percentuais das variáveis categóricas e numéricas.

O estudo teve aprovação no Comitê de Ética em Pesquisa do Hospital Eduardo de Menezes/HEM/FHEMIG com parecer de $\mathrm{n}^{\circ}$. 1.353.498. A pesquisa obedeceu aos critérios da Resolução n ${ }^{\circ}$. 466/2012 que regulamenta a pesquisa envolvendo seres humanos.

\section{RESULTADOS}

Participaram da pesquisa $43(63,23 \%)$ ACS ativos no município, os demais não foram inclusos por se recusarem a participar ou por não estarem presentes no momento da coleta. Quanto ao gênero $32(74,41 \%)$ eram do sexo feminino e $11(25,58 \%)$ do sexo masculino. Entre estes ACS, $17(39,53 \%)$ relataram que possuíam e ou possuem pessoas com diagnóstico de Hanseníase em sua microrregião. Os resultados foram categorizados, para análise, em 4 blocos: Bloco 1- A doença (aspectos gerais), Bloco 2- Diagnóstico, Bloco 3- Transmissão, Bloco 4- Tratamento.

No que diz respeito ao bloco 1 sobre os aspectos gerais, a maioria dos ACS reconheceu a doença como sendo contagiosa $(79,06 \%, \mathrm{n}=34)$ e infecciosa $(62,79 \%, \mathrm{n}=27)$, que atinge a pele $(97,67 \%, \mathrm{n}=42)$ e nervos $(74,41 \%, \mathrm{n}=32)$. Houve divergências quanto aos reconhecimentos dos sinais e sintomas, sendo que $58,13 \%(\mathrm{n}=25)$ apontaram como principal sintoma o aparecimento de coceira e vermelhidão no local afetado, enquanto $83,72 \%(n=36)$ destacaram o aparecimento de lesões insensíveis e de vários tipos.

No caso dos principais métodos de diagnóstico, a maioria listou exames laboratoriais $(83,73 \%, n=35)$ e diagnóstico clínico das lesões $(86,04 \%, \mathrm{n}=37)$. No total de $83,72 \%(\mathrm{n}=36)$ reconheceram a importância do diagnóstico para o tratamento da doença.

No quesito transmissão, $67,44 \%(n=29)$ destacaram que esta poderia ser feita por meio de gotículas respiratórias 


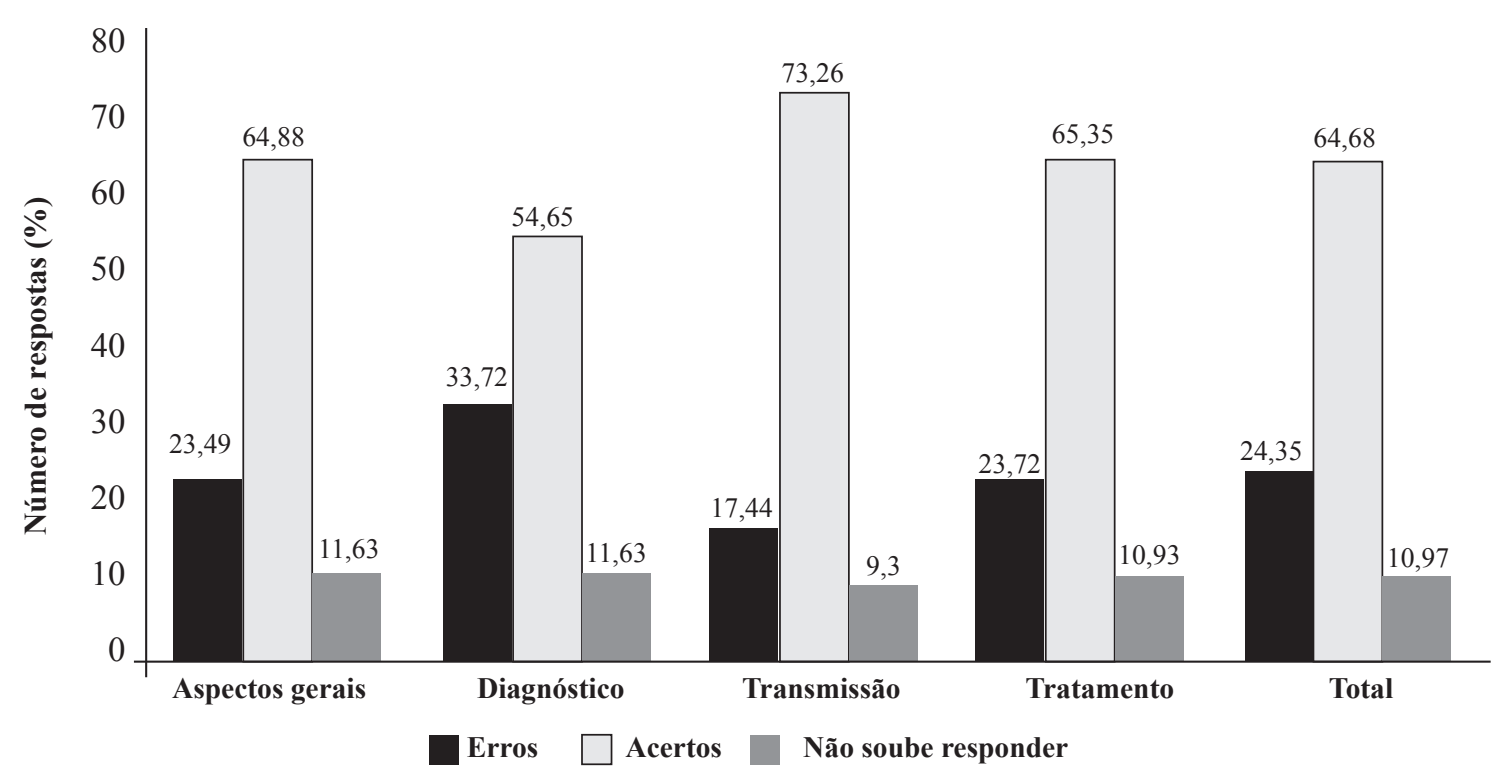

Figura 1 - Nível de informação dos Agentes Comunitários de Saúde sobre hanseníase. Cocal, Piauí, 2016.

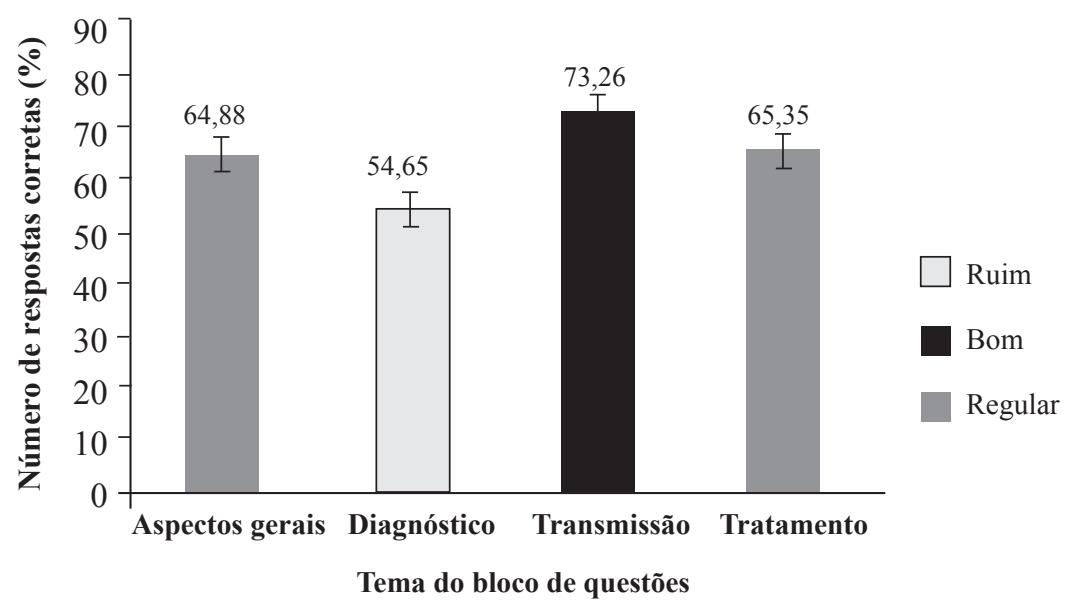

Figura 2 - Avaliação do nível de informação sobre hanseníase dos Agentes Comunitários de Saúde. Cocal, Piauí, 2016.

de pacientes não tratados; $86,04 \%(n=37)$ reconheceram que pessoas sem o tratamento são fontes de transmissão; $95,34 \%(n=41)$ afirmaram que o agente infeccioso para de ser transmitido logo após o início do tratamento.

Em relação ao tratamento, o uso de anti-inflamatórios isolados $(39,53 \%, n=19)$ foi a terapia mais associada. Quase a totalidade dos ACS afirmou que o tratamento deve ser realizado até o final, mesmo com o desaparecimento dos sintomas $(88,37 \%, \mathrm{n}=38)$ e de modo supervisionado $(93,02 \%, n=40)$. O tratamento correto foi associado com diminuição de recidivas $(72,09 \%, \mathrm{n}=31)$ e sequelas $(90,69 \%, n=39)$. A cura foi reconhecida por $97,67 \%(n=42)$ dos profissionais.

O panorama sobre erros e acertos de cada bloco pode ser visualizado na figura 1 .
Os resultados apontam um nível de conhecimento "bom" para o bloco 3 (Transmissão), "regular" para os blocos 1 (Aspectos gerais) e 4 (Tratamento) e "ruim" para o bloco 2 (Diagnóstico), como mostra a figura 2.

Quando analisado o panorama geral, o nível de informação dos ACS, no total de acertos do questionário, mostrou-se regular, alcançado uma porcentagem de 64,68\% $(\mathrm{n}=887)$.

\section{DISCUSSÃO}

O profissional ACS é um importante mediador entre a comunidade e a equipe de saúde. Sua atuação na disseminação de informações entre os indivíduos sob sua responsabilidade foi objeto de estudo em se tratando de 
diversas patologias ${ }^{(17,18)}$. Dentre as atribuições do ACS, destacam-se a realização de visita domiciliar e a busca ativa de casos de doenças prevalentes ${ }^{(14,15,20)}$.

Neste quesito, faz-se necessário que este profissional possua um conhecimento adequado sobre características, sinais, sintomas e tratamento das patologias mais recorrentes. O ACS apresenta-se como fundamental na deteç̧ão de casos de hanseníase, doença que deve ser identificada precocemente por apresentar caráter lesivo e incapacitante ${ }^{(4,6,14)}$.

Não foram encontrados estudos que verificassem o impacto do nível de informação do ACS na redução de índices de patologias. Porém, acredita-se na existência de uma cadeia de informações, onde o ACS em posse de subsídios adequados leva conhecimento à comunidade, esta por sua vez passa a deter informação relevante e colaborar com a promoção de sua própria saúde ${ }^{(21)}$.

Observou-se que o nível de conhecimento dos ACS do município de Cocal - Piaú sobre os principais aspectos da hanseníase varia entre "bom", "regular" e "ruim". Sendo considerado regular quando analisado o panorama geral $(64,68 \%)$.

Os ACS entrevistados obtiveram uma média de $73,26 \%$ de acertos quando questionados sobre a transmissão da hanseníase. O conhecimento acerca desse tópico foi considerado "bom" por estar entre 70 e $79 \%$ de acertos. Os principais mecanismos de transmissão do agente infeccioso causador da hanseníase também foram identificados pela maioria dos ACS entrevistados. Uma pesquisa realizada em Teresina, Piauí, confirma esses achados em que os ACS também apontaram um bom conhecimento sobre a temática transmissão ${ }^{(22)}$

Os ACS entrevistados neste estudo apresentaram um perfil de conhecimento classificado como "regular" no que diz respeito aos aspectos gerais da doença, cuja média de acertos ficou entre 60 e $69 \%$. Nesse bloco de perguntas, foram abordados itens como caráter contagioso e infeccioso da doença, sítios anatômicos afetados e os principais sinais e sintomas da hanseníase.

Um perfil semelhante foi encontrado em um estudo realizado no interior da Paraíba em que os ACS apresentaram compreensão limitada acerca de aspectos básicos e manifestações clínicas da hanseníase ${ }^{(13)}$. Em contrapartida, outro estudo relatou que todos os ACS entrevistados reconheceram os principais sinais e sintomas da hanseníase, assim como as características básicas da doença, classificando o conhecimento desses profissionais como muito satisfatório ${ }^{(22)}$.

O bloco "tratamento" apresentou perfil semelhante ao bloco "aspectos gerais", onde os ACS entrevistados apresentaram um perfil de conhecimento classificado como "regular". Nesse bloco de perguntas foram abordados itens como esquemas terapêuticos, sequelas, recidiva e cura da doença.

No estudo realizado na Paraíba, os ACS também encontraram dificuldades para identificar ações para o controle e o tratamento da hanseníase, embora o esquema terapêutico utilizado esteja bem claro para a maioria dos profissionais, principalmente para a forma multibacilar, assim como a cura por meio do tratamento ${ }^{(13)}$. No que diz respeito às medidas de tratamento e prevenção, os ACS de Teresina apresentaram uma atuação aquém do esperado ${ }^{(20)}$. Em contrapartida, todos os ACS de Teresina reconheceram ou citaram pelo menos um aspecto importante sobre o tratamento da doença, fazendo com que o conhecimento acerca do tratamento da hanseníase tivesse destaque positivo $^{(22)}$.

A maioria dos ACS entrevistados nesta pesquisa reconheceu a cura da hanseníase por meio do tratamento correto, completo e supervisionado. O percentual de compreensão sobre a cura da hanseníase associada ao tratamento se assemelha ao encontrado por outros autores ${ }^{(13)}$, em que $97,7 \%$ reconheceu a possibilidade de cura para a doença.

A hanseníase tem cura, no entanto, se não diagnosticada e tratada cedo pode trazer graves consequências para os portadores e seus familiares, pelas lesões que os incapacitam fisicamente. A adesão ao tratamento e sua realização de maneira correta são fundamentais para a eliminação da doença, com esse, torna-se possível, eliminar a transmissão, fechar a fonte de infecção e reduzir o risco de sequelas, sendo, portanto, ferramenta estratégica para o manejo da hanseníase como problema de saúde pública ${ }^{(23)}$.

$\mathrm{O}$ que se ressalta como um quesito importante para o seguimento e adesão à Poliquimioterapia (PQT), pois, em sua atuação, o ACS pode orientar o paciente durante o tratamento e reafirmar a possibilidade de cura da doença. Já que, apesar da divulgação na mídia e da difusão das políticas de atenção à hanseníase, a crença na cura da doença não é completa e afeta negativamente a adesão ao tratamento ${ }^{(24,25)}$.

A importância da informação dos profissionais ACS sobre a terapêutica utilizada pode refletir nas ações de controle da patologia, visto que a adesão ou abandono, as ações de autocuidado, casos de recidivas ou reações hansênicas podem ser muitas vezes identificados por este membro da ESF que está em contato mais capilar com a comunidade $^{(13)}$.

O pior desempenho dos ACS foi acerca do diagnóstico da hanseníase. A média de acerto desse bloco ficou entre 50 e $59 \%$, classificando o conhecimento acerca dos métodos de identificação e confirmação diagnóstica, classificação operacional baseada no diagnóstico e diagnóstico diferencial, como "ruim". 
Aponta-se, a partir da investigação sobre o processo de formação e capacitação dos ACS do referido município, e infere-se sobre alguns fatores que podem estar influenciando o nível de conhecimento desses profissionais. Destaca-se o grau de instrução nível médio e a não apropriação das atividades do cargo, decorrente da deficiência na orientação e atualização para exercer a função. Ademais, nenhum ACS entrevistado relatou ter participado de um curso de atualização em hanseníase. O conhecimento sobre a doença foi adquirido apenas durante o curso de formação profissional.

Esse problema não parece ser específico dessa população. Numa pesquisa realizada em quatro estados das regiões Norte e Nordeste (Tocantins, Pará, Piauí e Maranhão), apenas metade dos ACS fez um curso específico de hanseníase, entretanto com carga horária igual ou inferior a 8 horas, sendo o mais recente dois anos antes da entrevista(26).

A carência de cursos de capacitação e atualização junto a esses profissionais é corroborada pela literatura ${ }^{(13,20)}$. O conhecimento limitado envolvendo questões básicas da doença pode prejudicar a abordagem do ACS junto ao paciente. A educação em saúde de forma constante e direcionada se faz necessária uma vez que a existência de conhecimentos incorretos ou até mesmo precários vem a interferir no melhor atendimento à população, implicando em atitudes contrárias ao Programa Nacional de Controle da Hanseníase ${ }^{(18,22)}$.

Sabe-se que a educação em saúde em torno da hanseníase ainda é uma dificuldade que envolve questões como a identificação dos sinais e dos sintomas da patologia, ações de tratamento, de controle e de cura da doença. Assim, o processo de capacitação e treinamento dos ACS é apontado como ferramenta de destaque a fim de desenvolver habilidades eficientes a serem implementadas na sua rotina laboral ${ }^{(13)}$. A orientação/informação veiculada pelo ACS é uma atribuição primordial em seu processo de trabalho, deste modo à educação permanente desse sujeito deve respeitar as singularidades do perfil deste trabalhador. No entanto, as capacitações promovidas ainda são incipientes em frente à demanda dos $\mathrm{ACS}^{(13,17,18,22)}$.

Dessa forma, fica mais que claro a necessidade de cursos de treinamento/capacitação/atualização sobre hanseníase para esses profissionais, ressaltando a forma de abordagem. Sendo a profissão ACS um cargo que requer nível médio de escolaridade, ressalta-se a importância da linguagem ser direcionada e utilizada de acordo com o grau de instrução da maioria desses profissionais. Além disso, a forma de abordagem do conteúdo deve ser clara, direta, específica e direcionada para a realidade desses profissionais. Pesquisadores ${ }^{(27)}$ observaram a importância dessa abordagem diferenciada e desenvolveram, por meio de grupos, ações de educação em saúde com ACS no Rio Grande do Sul, focalizando a temática hanseníase. Neste sentido, o projeto permitiu visualizar, por meio da ferramenta dos grupos educativos, uma prática que prioriza as necessidades dos ACS em estar em constante aprendizado. Esta prática permitiu que os ACS tivessem um olhar mais crítico em relação à doença e seus sintomas.

O estudo teve como limitações a adoção de linguagem no questionário que viesse a contemplar o nível de instrução dos ACS, muitas vezes não tendo como fugir da linguagem técnica que dificultava a compreensão deste profissional de questões referentes à patologia, diagnóstico, transmissão e tratamento da hanseníase. Sugere-se maiores estudos, que analisem o impacto de educação permanente no nível de informação do profissional ACS.

\section{CONCLUSÃO}

Os resultados obtidos nesta pesquisa evidenciaram um nível de informação aquém do esperado para os ACS de Cocal - Piauí, apresentando nível de conhecimento considerado "regular" sobre os principais aspectos da hanseníase analisando o panorama geral. Fazendo-se necessário que maior atenção seja dada na educação permanente em saúde desse profissional, promovendo estratégias que possam minimizar esse déficit, como grupos de discussão ou cursos específicos.

\section{AGRADECIMENTOS}

Os pesquisadores agradecem a cordialidade $\mathrm{e}$ disponibilidade da Secretaria Municipal de Saúde de CocalPI.

\section{REFERÊNCIAS}

1. Ministério da Saúde (BR), Secretaria de Vigilância em Saúde, Departamento de Vigilância Epidemiológica. Doenças infecciosas e parasitárias: guia de bolso. $8^{\mathrm{a}} \mathrm{ed}$. rev. Brasília: Ministério da Saúde; 2010.

2. Portal da Sociedade Brasileira de Dermatologia. Hanseníase [acesso em 2016 Mar 16]. Disponível em: http://www.sbd.org.br/doencas/hanseniase/

3. Ribeiro FS, Silva MLA, Mendonça ALB, Soares JSA, Freitas CSL, Linhares MSC. Qualidades dos serviços prestados pelos centros de saúde da família de SobralCeará aos portadores de hanseníase nos anos de 20092010. Sanare (Sobral). 2012;11(2):44-51.

4. Organização Mundial da Saúde - OMS. Eliminação da hanseníase [acesso em 2016 Mar 7]. Disponível em: http://www.who.int/lep/en/\#teste. 
5. Organização Mundial da Saúde - OMS. OMS Divulga situação mundial da hanseníase. [acesso em 2016 Mar 7]. Disponível em: http:/ www.paho.org/bra/index.php?option $=$ com content\&view $=$ article\&id=1477:oms-divulgasituacao-mundial-hanseniase $\&$ Itemid $=777$.

6. Sociedade Brasileira de Hanseneologia. Todos contra a hanseníase. [acesso em 2016 mar 25]. Disponível em: http://www.sbhansenologia.org.br/

7. Ministério da Saúde (BR), Secretaria de Vigilância em Saúde, Departamento de Vigilância Epidemiológica, Programa Nacional de Eliminação da Hanseníase. Plano Nacional de Eliminação da hanseníase em Nível Municipal, 2006-2010. Brasília: Ministério da Saúde; 2006.

8. Ignotti E, Andrade VLG, Sabroza PC, Araújo AJG. Estudo da adesão ao tratamento da hanseníase no município de Duque de Caxias Rio de Janeiro: abandono ou abandonados. Hansen Inc. 2001;26(11):23-30.

9. Ignotti E, Rodrigues AM, Andrade VLG, Valente JG. Aplicação de métodos de estimativa e prevalência de hanseníase no estado de Mato Grosso. Rev Bras Epidemiol. 2004;7(2):155-66.

10. Oliveira DC, Sá CP, Gomes AMT, Ramos RS, Pereira NA, Santos WCR. A política pública de saúde brasileira: representação e memória social de profissionais. Cad Saúde Pública. 2008; 24(1):197-206.

11. Oliveira VM, Assis CRD, Silva KCC. Levantamento epidemiológico da hanseníase no nordeste brasileiro durante o período de 2001-2010. Scire Salutis. 2013; 3(1):16-27.

12. Ximenes FRG Neto, Martins FR, Liberato BTG, Carvalho JP Filho, Aguiar BEM, Martins AR. Ações de sustentabilidade para o controle da hanseníase: a experiência do município Cariré - Ceará. Sanare (Sobral). 2011;10(2):71-4.

13. Andrade CG, Costa ICP, Freire MEM, Santos KFO, Gouveia EML, Claudino HG. Hanseníase: compreensão de agentes comunitários de saúde. Rev Bras Ciênc Saúde. 2011;15(1):17-24.

14. Ministério da Saúde (BR), Secretaria de Atenção à Saúde, Departamento de Atenção Básica. Vigilância em saúde: Dengue, Esquistossomose, Hanseníase, Malária, Tracoma e Tuberculose. $2^{\mathrm{a}}$ ed. Brasília: Ministério da Saúde; 2008.

15. Carvalho R Filho, Santos SS, Pinto NMM. Hanseníase: detecção precoce pelo enfermeiro na atenção primária. Rev Enfermagem Integrada. 2010; 3(2):606-20.

16. Ribeiro MDA, Bezerra EMA, Silva JCA, Campelo
GO, Freitas CASL. A visão do Agente Comunitário de Saúde (ACS) acerca do serviço de fisioterapia no núcleo de apoio à saúde da família (NASF) em Parnaíba, Piauí. Sanare (Sobral). 2013;12(2):14-20.

17. Araujo MRN, Assunção RS. A atuação do agente comunitário de saúde na promoção da saúde e na prevenção de doenças. Rev Bras Enferm. 2004; 57(1):19-25.

18. Trapé CA, Soares CB, Dalmaso ASW. Trabalho do agente comunitário de saúde: a dimensão educativa da supervisão. Sociedade Debate. 2011;17(1):119-38.

19. Secretaria Municipal de Saúde de Cocal - Piauí. Equipes de saúde do município de Cocal-PI [acesso em 2016 Mar 21]. Disponível em: cocal.pi.gov.br

20. Araujo DYML, Andrade JS, Madeira MZA. A atuação dos agentes comunitários de saúde do município de Teresina/Piauí sobre hanseníase. Rev RENE. 2011;12(N. esp.):995-1002.

21. Ávila MMM. Origem e evolução do programa agentes comunitários de saúde no Ceará. Rev Bras Promoç Saúde. 2011;24(2):159-68.

22. Sales JCS, Luz VLES, Castelo-Branco FMF, Araujo MFF, Castro SHTS, Silva TCA. O significado da hanseníase para o agente comunitário de saúde. Rev Interd. 2013;6(1):17-24.

23. Ministério da Saúde (BR), Departamento de Atenção Básica. Guia para o Controle da hanseníase. Brasília: Ministério da Saúde; 2002.

24. Lins AUFA. Representações sociais e hanseníase em São Domingos do Capim: um estudo de caso na Amazônia. Physis (Rio de J). 2010;20(1):171-94.

25. Queiroz MS, Carrasco MAP. O doente de hanseníase em campinas: uma perspectiva antropológica. Cad Saúde Pública. 1995;11(3):479-90.

26. Alencar OM, Heukelbach J, Pereira TM, Barbosa JC. Trabalho do agente comunitário de saúde no controle da hanseníase. Rev RENE. 2012;13(1):103-13.

27. Ceretta DR, Rotoli A, Cargnin MCS, Aires M. Grupo de educação em saúde como ferramenta de trabalho com agentes comunitários de saúde: prevenção da hanseníase. Rev Enfermagem. 2012; 8(8):208-17.

\section{Endereço para correspondência:}

Jefferson Carlos Araujo Silva

Universidade Federal do Maranhão - UFMA

Hospital Universitário Presidente Dutra

Rua Barão de Itapary, 227

Bairro: Centro

CEP: 65020-070 - São Luís - MA - Brasil

E-mail: jeffcasilva@gmail.com 